\title{
Thrombosed Abdominal Aortic Aneurysm in a Young Female
}

\author{
Sheshidhar Madaka ${ }^{1}$ \\ ${ }^{1}$ Nizam's Institute of Medical Sciences, Hyderabad, Telangana, India \\ Ind J Car Dis Wom 2021;6:209-215.
}

Presenter: A 35-year-old female, with the following chief complaints:

- Shortness of breath (SOB) since 7 days and chest pain since 4 days.

- Patient was apparently asymptomatic 7 days back; thereafter, she developed SOB, which was sudden onset, and New York Heart Association functional class (NYHA FC) III, which progressed to FC IV over the next 2 days and was also associated with orthopnea. Patient had no history of paroxysmal nocturnal dyspnea (PND) episodes.

- Chest pain-one episode 4 days back, sudden onset, retrosternal, squeezing type, associated with sweating, continued for 30 minutes, and was relieved with medication.

- Patient did not have any history of palpitations, syncope, bilateral swelling of legs, reduced urine output, facial puffiness, fever, cough, abdominal pain or distension, vomiting, diarrhea, back pain, skin rash, weight loss, easy fatigability, joint pain, ulcerations in the body, and history suggestive of rheumatic disease in past.

- Obstetric history: She had a past history of four spontaneous abortions as well as a history of surgery for ovarian cyst (8 years back). She conceived with in vitro fertilization (IVF) but spontaneously aborted.

- Patient had no similar complaints in the past. She is a homemaker. Patient takes mixed diet. She is not a known smoker, alcoholic, or tobacco chewer. Patient is not using any other medication. Family history was not suggestive of any cardiac disease or other medical illnesses in her family.

Discussant: To summarize the clinical details, a 35-year-old female presented with sudden onset of dyspnea since 7 days, progressed from class III to IV in 2 days, one episode of chest
Address for correspondence Sheshidhar Madaka, Nizam's Institute of Medical Sciences, Hyderabad, Telangana, 500082, India (e-mail: sheshidharmadaka@gmail.com).

pain 4 days back, and past history of abortions. Based on clinical presentation, the differential diagnoses included the following:

Antiphospholipid antibodies (APLA) syndrome: These patients presented with vascular thrombotic events in the form of deep vein thrombosis (DVT), acute pulmonary thromboembolism, acute coronary syndrome (ACS) due to thrombotic occlusion of coronaries, and recurrent abortions; hence, the possibility of APLA syndrome can be considered as first possible diagnosis. ${ }^{1}$

Systemic lupus erythematosus (SLE): SLE patients presented with fever, joint pains, myalgias, skin rashes, cardiac involvement in the form of pericarditis and myocarditis. So, these patients can present as SOB and chest pain; however, no prodromal symptoms were seen in the patient.

ACS: Patients diagnosed with ACS manifest with chest pain and dyspnea if the patient developed left ventricular (LV) dysfunction, and they were also predisposed to develop arrhythmias. In our patient, coronary artery disease (CAD) can be considered. ${ }^{2}$

Dilated cardiomyopathy (DCMP): Patients diagnosed with DCMP usually manifest with SOB, which is usually insidious in onset and gradually progressive, and atypical chest pain. Patients diagnosed with DCMP will usually have long duration of symptoms; however present patient had breathlessness of only 7 days duration.

Viral myocarditis: Patients diagnosed with viral myocarditis usually have SOB, which is sudden in onset and includes chest pain. Hence, in this patient, there is high possibility of viral myocarditis. However, this patient did not elicit any inciting event.

Hypertrophic cardiomyopathy (HCM): Patients diagnosed with HCM usually develop symptoms by the 3rd and 4th
DOI https://doi.org/ 10.1055/s-0041-1736252. ISSN 2455-7854.
(C) 2021. Women in Cardiology and Related Sciences. All rights reserved.

This is an open access article published by Thieme under the terms of the Creative Commons Attribution-NonDerivative-NonCommercial-License, permitting copying and reproduction so long as the original work is given appropriate credit. Contents may not be used for commercial purposes, or adapted, remixed, transformed or built upon. (https://creativecommons.org/ licenses/by-nc-nd/4.0/)

Thieme Medical and Scientific Publishers Pvt. Ltd., A-12, 2nd Floor, Sector 2, Noida-201301 UP, India 
decade. ${ }^{3}$ HCM patients present with chest pain, syncope, palpitations, and SOB. ${ }^{4} \mathrm{HCM}$ patients present as dilated cardiomyopathy in the late burnt-out stages. About 60\% patients usually have a positive family history of cardiomyopathy. ${ }^{5}$ In the present case, family history was absent, and patient had sudden onset of symptoms.

Infiltrative cardiomyopathy: Patients diagnosed with infiltrative cardiomyopathy usually present with SOB and chest pain after 6th decade. Infiltrative cardiomyopathy usually manifest as a component of multisystem disorder. ${ }^{6}$ In our present patient, there were no clinical manifestations related to other systems.

Calcific degeneration of bicuspid aortic valve: Patients with significant aortic stenosis (AS) due to calcific degeneration of bicuspid aortic valve present with angina, syncope, and SOB. ${ }^{7}$ These patients have a slightly longer history of symptoms. Our patient had sudden onset of SOB and chest pain and no history of syncope and palpitations.

Presenter: On general physical examination, patient was conscious, coherent, and oriented. Her body mass index (BMI) was $23 \mathrm{~kg} / \mathrm{m}^{2}$ and temperature $98.4^{\circ} \mathrm{F}$. Pulse rate was 120 beats/min, and regular blood pressure was $190 / 100 \mathrm{~mm} \mathrm{Hg}$ in right arm supine position and 200/100 in right lower limb. Respiratory rate was 34 cycles per minute and oxygen saturation was $99 \%$ on room air. Pallor was present, and there were no signs of icterus, cyanosis, clubbing, edema feet or lymphadenopathy. On cardiovascular (CVS) examination, jugular venous pressure (JVP) had normal mean column height and normal wave pattern. Apex beat was localized in left 5 th intercostal space half inch medial to midclavicular line, LV type. First heart sound was normal in intensity. Second heart sound was normal in intensity and split. No added sounds were present. On auscultation of lungs, fine crackles were found. There was no evidence of organomegaly. No epigastric pulsations were noted on per abdomen examination. Central nervous system (CNS) examination was normal.

Discussant: Summary of the examination findings are blood pressure of 190/100 mm Hg in right arm supine position, pallor, and respiratory rate was 34 cycles per minute.

Keeping in mind history as well as examination findings, the probable differential diagnosis is:

Hypertensive heart failure: Presented with hypertension and cardiac involvement in the form of ischemic symptoms like chest pain and failure symptoms like SOB. Hypertensive heart disease can manifest as either systolic heart failure or diastolic failure or both. Hypertensive heart disease patients are more prone to develop acute events like decompensated heart failure, acute coronary events, or sudden cardiac death. ${ }^{8}$ Hypertension (HTN) disrupts the endothelial system, which increases the risk of CAD and peripheral arterial disease (PAD) and thus represents a significant risk factor for the development of atherosclerotic disease.

APLA syndrome: is an autoimmune disorder present as recurrent abortions and thromboembolic events like DVT, acute pulmonary thromboembolism and ACS. This patient has history of abortions, and acute coronary symptoms can be considered as initial differential diagnosis.

Autoimmune overlap syndromes: About 25 to 45 percent cases of APLA are associated with SLE. This can manifest as hypertension due to lupus nephritis, recurrent abortions and thrombotic events such as ACS, pallor due to hemolytic anemia, but other manifestations like skin involvement are absent in this case.

Early atherosclerosis: Early atherosclerosis can manifest as hypertension and acute coronary events. High blood pressure on its own increases atherosclerosis risk by 60 percent. Early atherosclerosis of renal and coronary vessels present as HTN and HTN-induced chronic kidney disease which manifests as anemia, renal failure and acute coronary events. Early atherosclerosis can be considered as initial diagnosis, but the female sex and age of presentation are against the atherosclerosis. ${ }^{9,10}$

Infiltrative cardiomyopathy: Infiltrative cardiomyopathy patients usually present with diastolic heart failure, concentric hypertrophy in initial stages, and in advanced stage, present as DCMP ${ }^{11}$ Occurrence of complete heart block and supraventricular tachyarrhythmia is high due to infiltration of conduction system and atrial dilatation. The typical features are absent in the present case.

Hypertensive HCM: Hypertension of mild-to-moderate degree sometimes occurs in patients with HCM, but it is predominant among the elderly. Early age of presentation in our case and anemia usually rules out this condition. ${ }^{12}$

\section{Investigations}

1. Chest X-ray

2. ECG

3. $2 \mathrm{D} \mathrm{ECHO}$

4. Laboratory investigations (-Table 1 )

5. Others

According to radiologist's opinion, in - Fig. 1, chest X-ray shows normal cardiac size with normal pulmonary vascular markings. Aorta and pulmonary bay are normal. No signs of pulmonary venous hypertension.

\section{ECG}

In -Fig. 2, ECG on the day of hospital admission was suggestive of normal sinus rhythm (NSR) rate of 96, left axis deviation, and T-wave inversions in lead $1 \mathrm{aVL}$ v3 to v6

In -Fig. 3, ECG after 1 hour suggestive of NSR with deepening of $\mathrm{T}$ inversions in lead $1 \mathrm{aVL}$ V3 to V6

ECG features suggestive of dynamic ECG changes. These features are in favor of ACS.

Discussant: In view of dynamic ECG changes, ACS is suspected, and patient is evaluated with troponin test and echocardiography.

\section{Troponin Test: Positive}

Echocardiography: normal-sized cardiac chambers, regional wall motion abnormalities (RWMA) + in distal interventricular 
Table 1 Laboratory investigations

\begin{tabular}{|l|l|}
\hline Parameters & Patient values \\
\hline $\mathrm{Hb}$ & 7.3 \\
\hline TLC & 7300 \\
\hline Platelets & 2.8 \\
\hline ESR & 140 \\
\hline $\mathrm{Na} / \mathrm{K}$ & $134 / 4.4$ \\
\hline Crt/BUL & $6 / 168$ \\
\hline Bil T/D & $0.2 / 0.1$ \\
\hline SGOT/PT & $13 / 11$ \\
\hline T prot/alb & $7.1 / 3.9$ \\
\hline LDH/CPK & $789 / 534$ \\
\hline NTproBNP & 17878 \\
\hline TC/TG/HDL/LDL & $120 / 185 / 40 / 73$ \\
\hline hsCRP & 24 \\
\hline Ca/Mg/PO4 & $8.5 / 0.78 / 4.9$ \\
\hline Uric acid & 9.2 \\
\hline Vit D/PTH & $26.48 / 440$ \\
\hline CUE & Prot 1+ \\
\hline Urine PCR & 1.8 \\
\hline
\end{tabular}

Abbreviations: ESR, erythrocyte sedimentation rate; HDL, high-density lipoprotein; hsCRP, high-sensitivity C-reactive protein; LDH, lactate dehydrogenase; LDL, low-density lipoprotein; PCR, protein to creatinine ratio; PTH, parathyroid; TC, total cholesterol; TC, triglyceride; TLC, total leucocyte count.

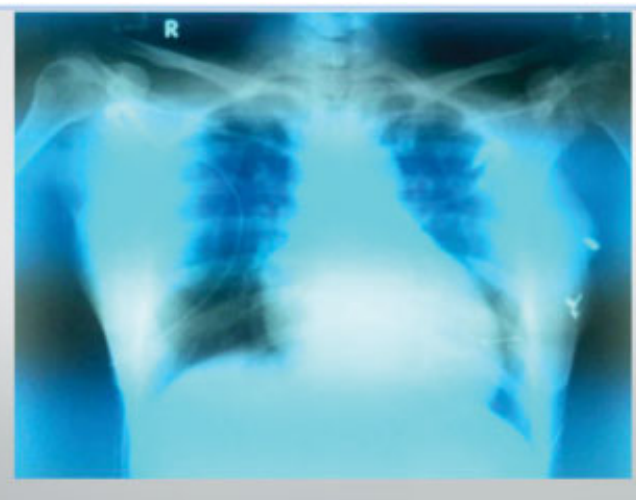

Fig. 1 Chest X-ray PA view.

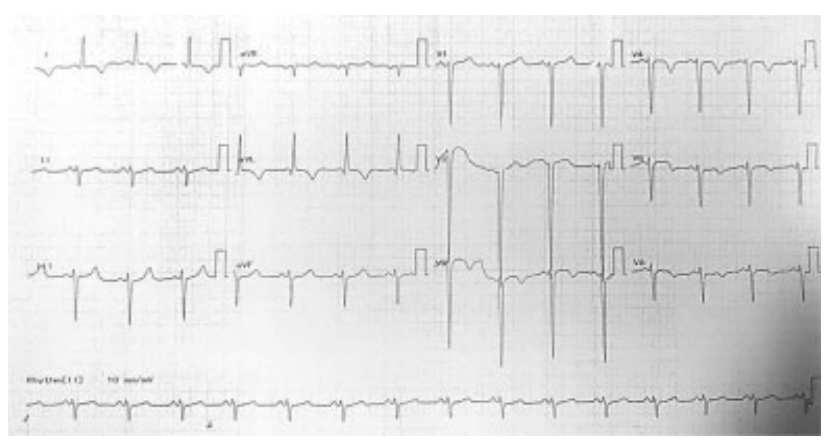

Fig. 2 ECG on hospital admission.

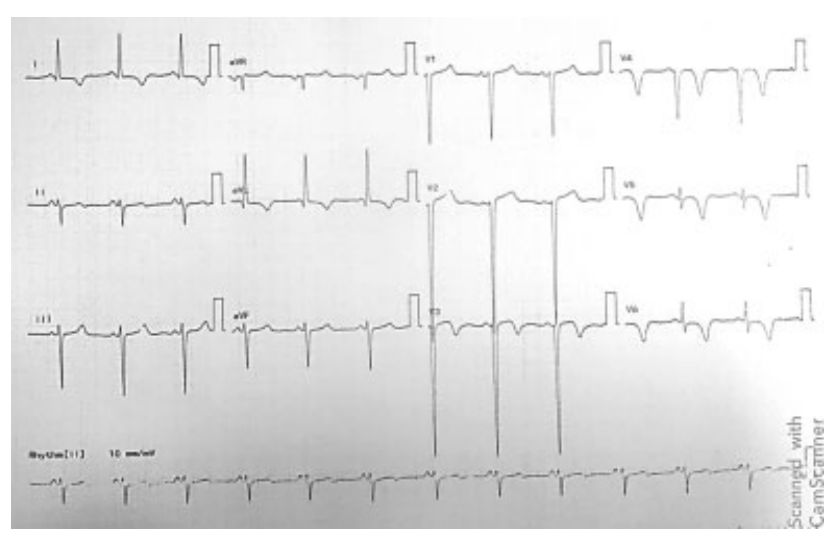

Fig. 3 ECG after 1 hour on hospital admission.

septum (IVS), apex, mild LV dysfunction (ejection fraction [EF]: $45 \%$ ), grade I diastolic dysfunction ( $\mathrm{A}>\mathrm{E}$ ), mild mitral regurgitation (MR) (MRJA 2.1SQCM) mild tricuspid regurgitation (TR), mild pulmonary arterial hypertension (PAH) (RVSP 36), good right ventricular (RV) function (TAPSE 2.1), no clot/vegetation/ pulmonary embolism (PE).

Discussant: Based on above ECG, echo changes patient had ACS non-ST-segment myocardial infarction (NSTEMI) with distal IVS and apex hypokinesia along with mild LV dysfunction.

Presenter: Patient was further evaluated with laboratory investigations ( shown in - Table 1)

Presenter: Above laboratory parameters ( - Table 1 ) suggest anemia with raised blood urea nitrogen (BUN) and creatinine values and increased NTproBNP and high-sensitivity C-reactive protein (hsCRP) values.

Discussant: Presence of normocytic normochromic anemia with raised renal parameters is more in favor of chronic kidney disease (CKD).

Patient was initially managed with diuretics and vasodilators. No significant improvement was found. Nephrology opinion was obtained, and hemodialysis done with blood transfusion. After stabilization, USG abdomen was done.

\section{USG Abdomen}

Radiologist opinion: showing bilateral (B/L) small kidneys, echogenicity, and loss of corticomedullary differentiation (CMD).

Right kidney: $43 \times 14 \mathrm{~mm}$.

Left kidney: $78 \times 33 \mathrm{~mm}$.

Abdominal aortic aneurysm extending from celiac axis till bifurcation. Maximum dimension is $54 \times 42 \mathrm{~mm}$.

There is concentric thrombus involving distal aorta with maximum thickness of $13 \mathrm{~mm}$ along with central lumen patent, extending into right common iliac artery (CIA).

- High-resolution (HRCT chest): mild cardiomegaly with pulmonary edema.

No significant lung parenchymal abnormality seen.

- COVID-19 RT-PCR: negative. 


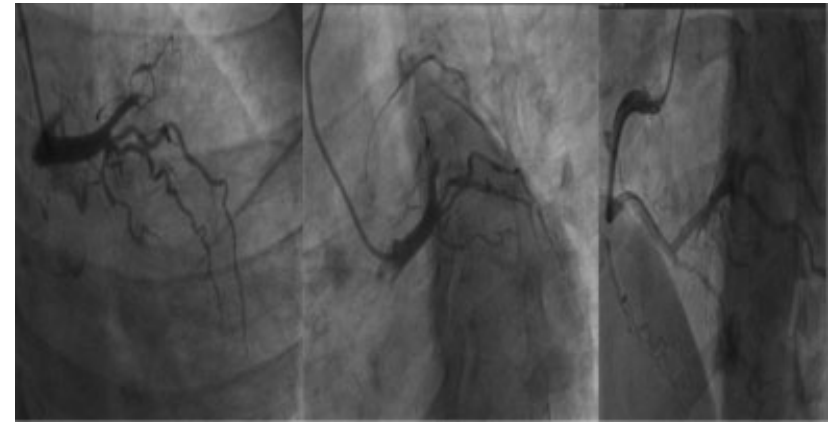

Fig. 4 Coronary angiogram (CAG) suggestive of triple-vessel disease.

\section{- Presenter:}

- Patient was subjected to one more session of hemodialysis with 1 unit packed red blood cell (PRBC) transfusion and coronary angiogram (CAG) done.

- CAG: suggestive of triple-vessel disease with thrombotic occlusion (-Fig. 4)

- Patient managed with percutaneous coronary intervention (PCI) to left anterior descending (LAD) artery (-Fig. 5).

Presenter: A 35-year-old female with history of recurrent abortions, de novo HTN with CKD, triple-vessel disease with thrombotic occlusion, and abdominal aortic aneurysm with thrombus.
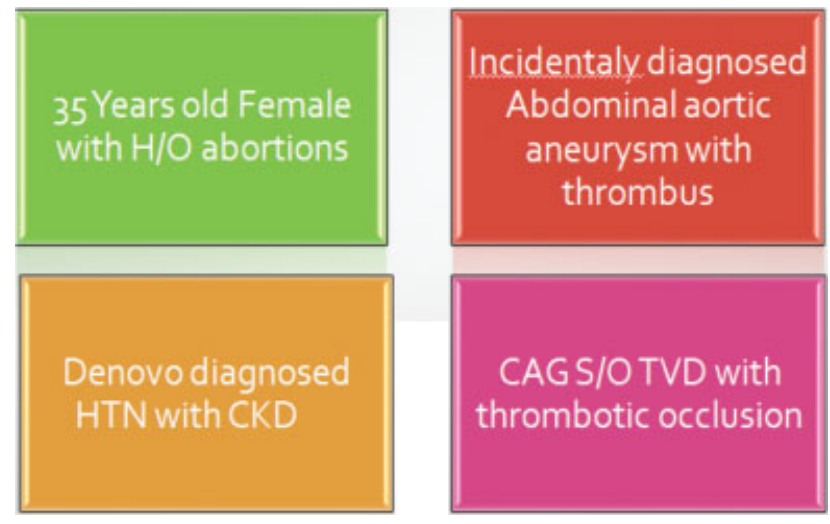

Discussant: Based on the above presentation, the differential diagnosis is as follows:

- APLA.

- Autoimmune overlap disorders.

- Large vessel vasculitis (Takayasu arteritis).

- Other vasculitic disorders.

- Behcet's disease.

- HTN-induced degenerative changes.

- Early atherosclerosis.

Presenter: APLA and autoimmune overlap disorders are considered as first possible diagnosis to confirm the diagnosis; patient is further investigated with autoimmune profile as shown in - Table 2.

Discussant: Patient has negative rheumatoid arthritis (RA) factor, lupus antibodies, $\beta 2$ glycoprotein, antinuclear antibody (ANA), ds DNA, complement factors, antihistone
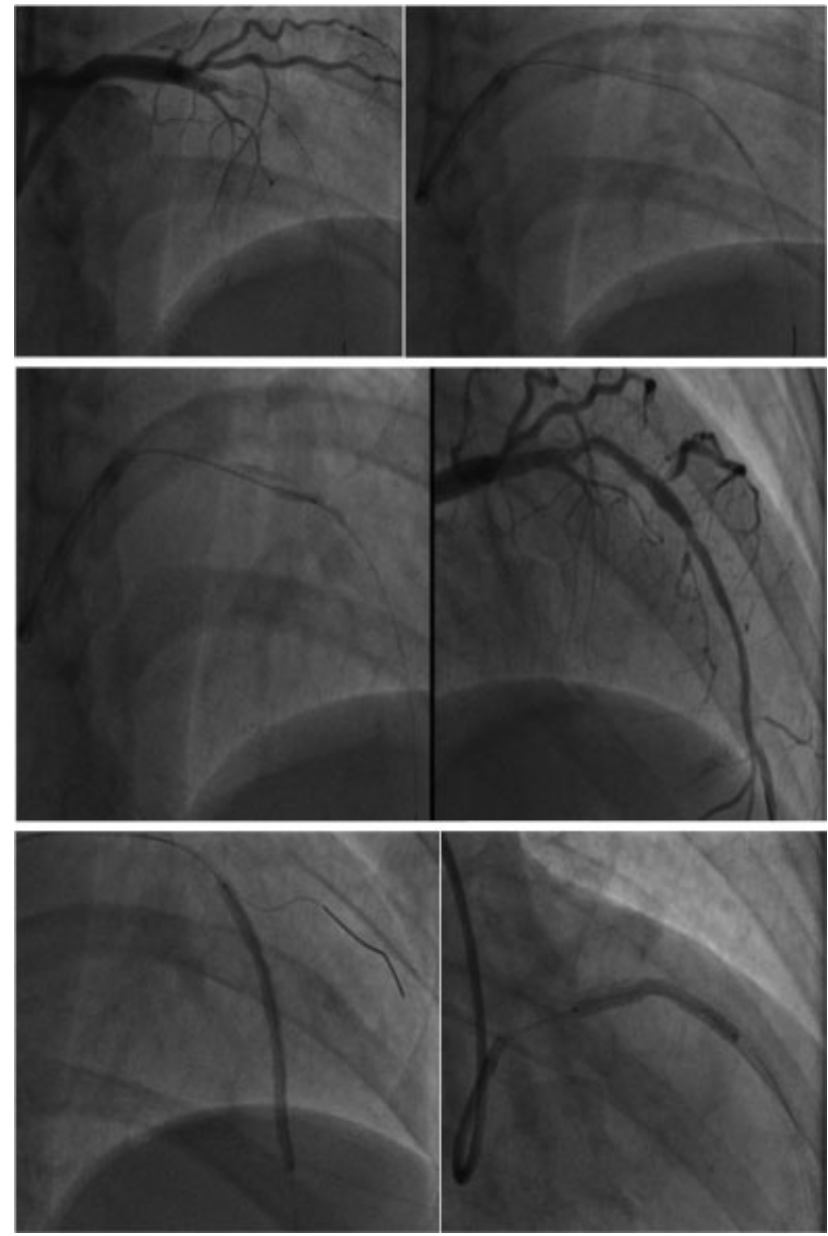

Fig. 5 Suggestive of percutaneous coronary intervention (PCI) to left anterior descending (LAD) artery.

antibodies, U1RNP, SS-A, SS-B, Scl-70, Jo-1, Mi-2, Ku, and CENP-B antibodies.

APLA syndrome: is a disease with a myriad of clinical manifestations ranging from recurrent vascular thrombosis and pregnancy complications to valvular lesions, transverse myelitis, thrombocytopenia and hemolytic anemia. It may be primary or secondary, that is, associated with other autoimmune diseases. The latest classification criteria (Sydney 2006) recognize just three tests to define this syndromelupus anticoagulant (LA), anticardiolipin antibodies and anti gp 1 antibodies. In our patient, these antibodies are negative.

Autoimmune overlap syndromes: APLA, which occurs secondary to SLE or other autoimmune diseases, is called secondary antiphospholipid antibody syndrome (APS) and is characterized by arterial and venous thrombosis, recurrent miscarriages in pregnancy, and neurologic manifestations along with the presence of APLA. APLA are more prevalent in patients with SLE $(\sim 50 \%)$ with LA constituting 15 to $34 \%$ and cardiolipin (CL) constituting 12 to $30 \%{ }^{13}$ In a study by Unikiene and Misiuniene, the incidence of APLA positivity was found to be $30.2 \%$ in the SLE group and $29 \%$ in the systemic sclerosis group. In our case, APLA antibodies, ds DNA, RA factor, ANA antibodies, SCL-70, SS-A, SS-B antibodies are negative. 
Table 2 Laboratory investigations

\begin{tabular}{|l|l|}
\hline Test & Result \\
\hline Anticardiolipin antibodies $(\mathrm{IgG}+\mathrm{IgM})$ & Negative \\
\hline B2 glycoprotein & Negative \\
\hline LA & Negative \\
\hline ANA & Negative \\
\hline Anti dsDNA & Negative \\
\hline RA factor & Negative \\
\hline Anti-CCP & Negative \\
\hline C3 & Normal \\
\hline C4 & Normal \\
\hline Test & RESULT \\
\hline Nucleosomes & Negative \\
\hline Histones & Negative \\
\hline SmD1 & Negative \\
\hline PCNA & Negative \\
\hline U1-snRNP & Negative \\
\hline SS-A/Ro52 & Negative \\
\hline SS-A/Ro60 & Negative \\
\hline SS-B/La & Negative \\
\hline SCl-70 & Negative \\
\hline AMA-M2 & Negative \\
\hline Jo-1 & Negative \\
\hline PM-Scl & Negative \\
\hline Mi-2 & Negative \\
\hline Ku & Negative \\
\hline CENP-B & Negative \\
\hline
\end{tabular}

Abbreviations: ANA, antinuclear antibody; CL, cardiolipin, LA, lupus anticoagulant; RA, rheumatoid arthritis.

\section{Mixed Connective Tissue Disorder}

It is a rare connective tissue disorder that is characterized by features commonly seen in three different connective tissue disorders: SLE, scleroderma, and polymyositis. Some affected people may also have symptoms of RA. ${ }^{14}$ Although mixed connective tissue disorder (MCTD) can affect people of all ages, it appears to be most common in women under age 30. Signs and symptoms vary but may include Raynaud's phenomenon, arthritis, heart, lung and skin abnormalities, kidney disease, muscle weakness, and dysfunction of the esophagus. In our case, classical manifestations are absent, and U1RNP antibodies are negative.

Presenter: After ruling out APLA and autoimmune overlap disorders, patient was further investigated with MR aortogram, as shown in - Fig. 6.

\section{MR AORTOGRAM}

\section{Radiologist Opinion}

- Ascending aorta, arch, and descending aorta are normal in course, caliber, and signal intensity

- Fusiform aneurismal dilatation of suprarenal, renal and infrarenal $(4.7 \mathrm{~cm})$ segments of abdominal aorta (AA) extending from D12-L1 vertebrae till bifurcation, and eccentric thrombus involving right proximal CIA measuring $14 \mathrm{~mm}$ till periphery

- Dilatation of right CIA with peripheral thrombus.

- Superior mesenteric artery (SMA) arising from aneurysmal segment of AA.

- Right RA arising from aneurysmal segment of AA, with narrowed distal portion and shrunken right kidney, as shown in - Fig. 7.

- Left RA is normal in caliber.

Presenter: Based on MRA findings, differential diagnoses considered in this case are as follows:

- Large vessel vasculitis (Takayasu arteritis).

- Behcet's disease.

- HTN-induced degenerative changes.
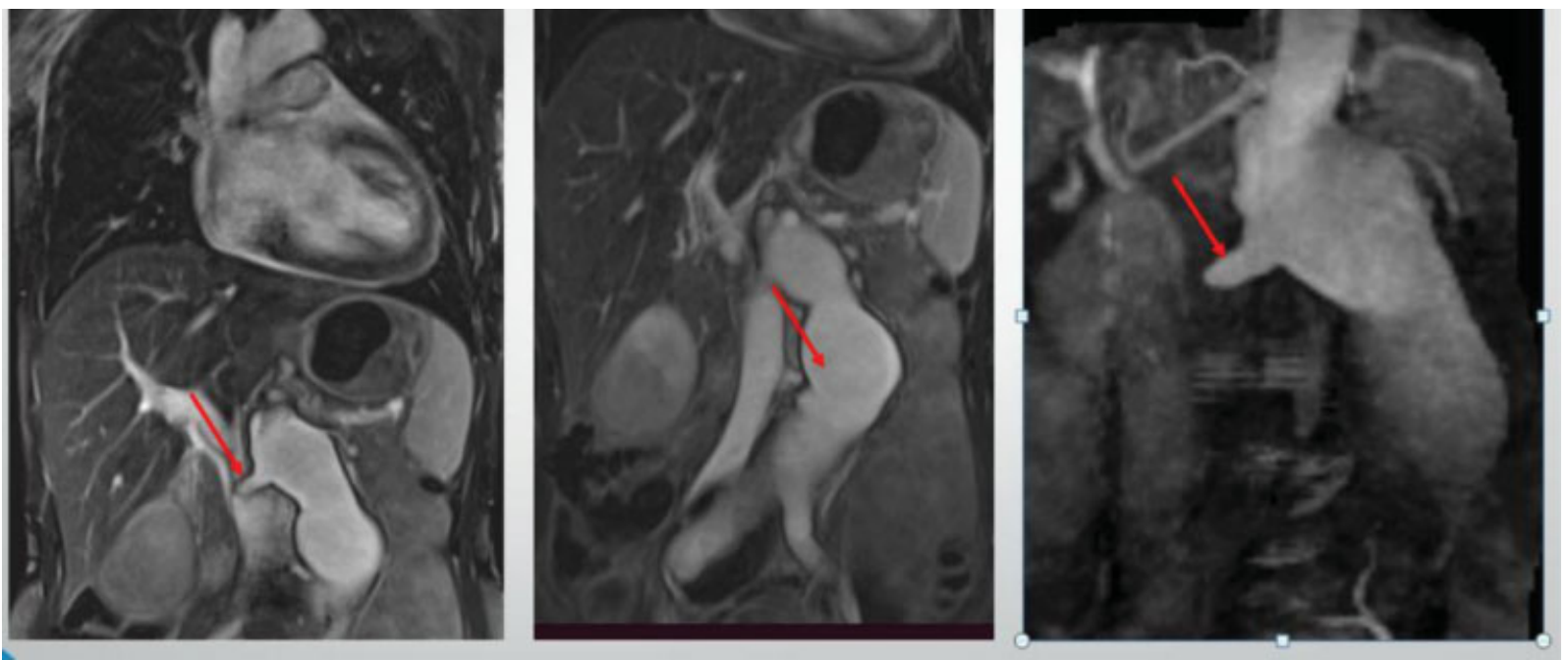

Fig. 6 MR aortogram showing fusiform aneurysmal dilatation of suprarenal, renal and infr-renal $(4.7 \mathrm{~cm})$ segments of abdominal aorta $(A A)$. 


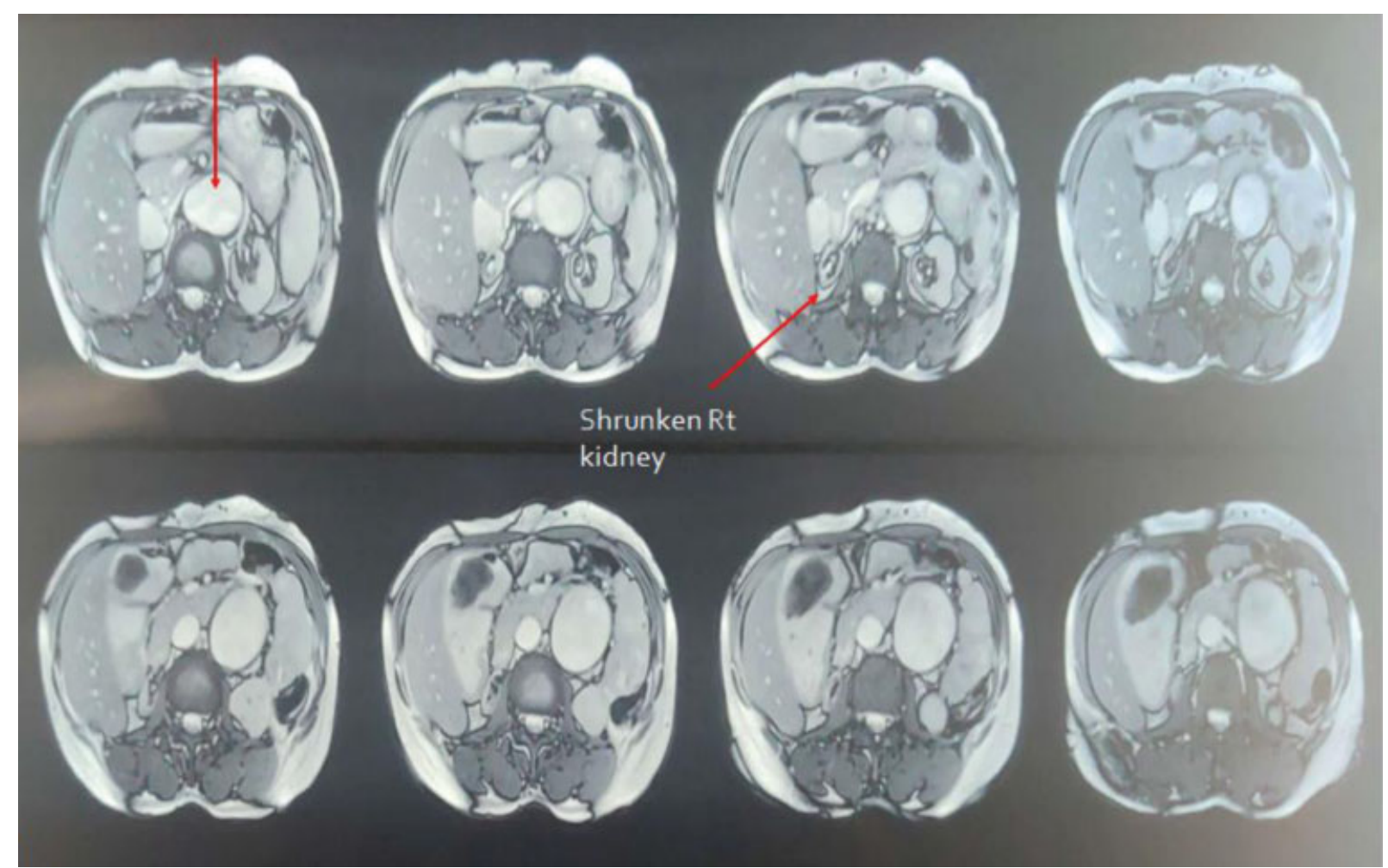

Fig. 7 MR aortogram showing shrunken right kidney.

\section{Early Atherosclerosis}

Large vessel vasculitis (Takayasu arteritis): is a rare systemic inflammatory vasculitis of large vessels with unknown etiology. It presents as granulomatous inflammation of aorta and its major branches and mainly involves carotid arteries and manifest as pulseless disease. In the acute phase of Takayasu arteritis, it results in thickening of vessel wall, mural edema and enhancement. The pulseless/occlusive phase, which results from vascular stenosis or occlusion and signs and symptoms, is dependent upon which vessels are involved. Stenosis is the most common complication of the aorta and most commonly involves the descending thoracic aorta and AA, although aortic occlusion or dilation may also occur. ${ }^{15}$ Most commonly involved vessels are subclavian arteries. Our case is not having classical features of Takayasu arteritis.

Behcet's disease: chronic relapsing remitting vasculitis affecting multiple organs present as recurrent oral ulcerations, genital ulcerations, uveitis, erythema nodosum vasculitis features like aneurysm, and positive pathergy test. ${ }^{16}$ In our case, classical manifestations are absent.

Presenter: After ruling out Takayasu arteritis and Behcet's disease, to differentiate HTN-induced degenerative changes and early atherosclerosis, patient was further probed with the following investigations, as shown in - Table 3.

Discussant: Patient has increased values of lipoprotein a, homocysteine, fibrinogen, and hsCRP.

HTM-induced degenerative changes: Abdominal aortic aneurysms (AAA) are fatal in $80 \%$ of the cases when ruptured. AAA in hypertensive versus nonhypertensive adult patients
Table 3 Atherosclerotic markers

\begin{tabular}{|l|l|}
\hline Test & Result (normal range) \\
\hline Lp (a) & $72(<30)$ \\
\hline Homocysteine & $53.52(<15)$ \\
\hline Fibrinogen & $720(200-400)$ \\
\hline hsCRP & 24 \\
\hline
\end{tabular}

Abbreviation: hsCRP, high-sensitivity C-reactive protein.

showed, overall, a $66 \%$ increased risk of developing the disease in hypertensive patients. ${ }^{17} \mathrm{HTN}$ has been considered a potential risk factor for AAA; furthermore, a $20 \mathrm{~mm} \mathrm{Hg}$ increase in systolic blood pressure (SBP) and a $10 \mathrm{~mm} \mathrm{Hg}$ increase in diastolic blood pressure (DBP) were associated with a $14 \%$ and $28 \%$ increase in the risk of developing AAA, respectively. In our case, patient had increased values of atherosclerotic markers.

Early atherosclerosis: Atherosclerosis in young women, although a relatively rare occurrence, needs close attention and vigorous treatment.

The risk factors for accelerated atherosclerosis in young women are HTN, smoking, oral contraceptive (OC) pills intake, hyperlipidemia, premature menopause, underlying inflammatory disease (e.g., SLE, RA), diabetes mellitus, and family history. Other possible risk factors have included prolonged steroid use, homocystinemia, thrombocytosis, blood clotting disorders, previous viral infections, hyperinsulinemia and, of course, genetic factors. 


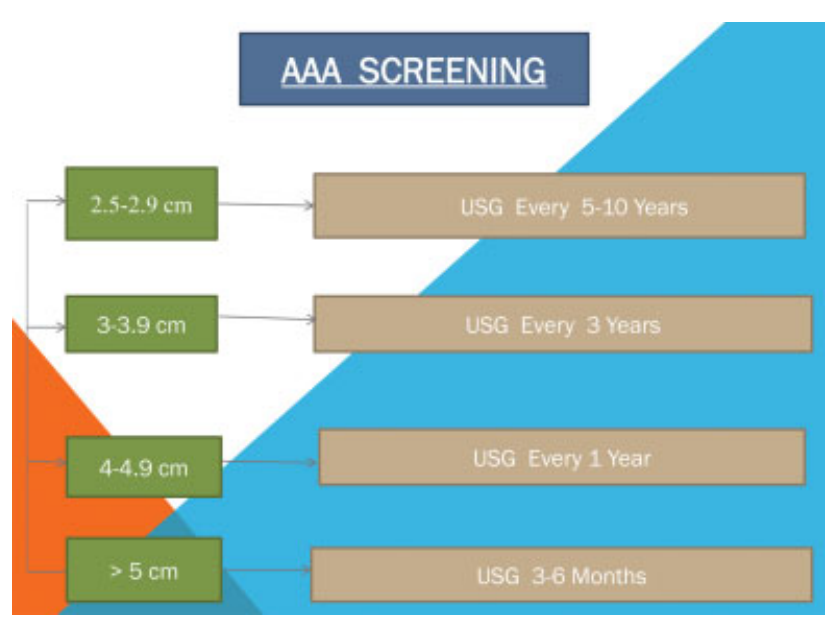

Fig. 8 Abdominal aortic aneurysm (AAA) screening guidelines.

Discussion: AAA is a disease predominantly seen in men $>50$ years, and it is more common in smokers. The incidence in women increases with age, with a prevalence of only 0.4 to $1.3 \%$ in women $>65$ years. The most common etiology seen is atherosclerosis, HTN, tobacco use, hypercholesterolemia, and associated CAD. The incidence in young women $<40$ years is reported to be $<0.1 \%$. The most common etiology seen is vasculitis, autoimmune disorders, early atherosclerosis associated with obesity, and dyslipidemia. In this case, patient presented with first episode of hypertensive emergency with de novo CKD, ACS (NSTEMI) and incidentally diagnosed AAA on imaging. The right renal artery is originating from the aneurysmal part of aorta with shrunken right kidney. So, renal failure in this case is due to AAA or AAA is due to renal failure and associated HTN, leading to degenerative changes which are not clear.

Also, the patient had high thrombus burden in coronaries as well as in AAA; CAD in young is usually seen in males with strong association with smoking. CAD in young females is again rarely seen and factors associated with this early atherosclerosis include CKD, HTN, dyslipidemia, South Asian race, homocysteinemia, Lp (a), metabolic syndrome, and other vasculitic or autoimmune causes.

In this case, renal failure with HTN can be considered as the factor triggering early atherosclerosis and CAD. All the inflammatory markers having strong association with early atherosclerosis were positive in this patient.

Clinical diagnosis: early atherosclerosis with ACS NSTEMI with AAA.

Discussion of management: Patient treated with diuretics, vasodilators, antiplatelets, statins, hemodialysis, blood transfusion, and PCI to LAD.

\section{Abdominal Aorta Screening}

AAA screening guidelines as shown in - Fig. 8.

Presenter: Patient is advised to follow-up with USG abdomen and pelvis every year, based on screening guidelines.

\section{Final Diagnosis}

Thrombosed AAA in a young female due to early atherosclerosis.

Conflict of interest

None declared.

\section{References}

1 Bidot CJ, Jy W, Horstman LL, Ahn ER, Yaniz M, Ahn YS. Antiphospholipid antibodies (APLA) in immune thrombocytopenic purpura (ITP) and antiphospholipid syndrome (APS). Am J Hematol 2006;81(06):391-396

2 Al-Absi HR, Refaee MA, Rehman AU, Islam MT, Belhaouari SB, Alam T. Risk factors and comorbidities associated to cardiovascular disease in Qatar: a machine learning based case-control study. IEEE Access 2021;9:29929-29941

3 Semsarian C, Ingles J, Maron MS, Maron BJ. New perspectives on the prevalence of hypertrophic cardiomyopathy. J Am Coll Cardiol 2015;65(12):1249-1254

4 Olivotto I, Cecchi F, Casey SA, Dolara A, Traverse JH, Maron BJ. Impact of atrial fibrillation on the clinical course of hypertrophic cardiomyopathy. Circulation 2001;104(21): 2517-2524

5 Marian AJ, Braunwald E. Hypertrophic cardiomyopathy: genetics, pathogenesis, clinical manifestations, diagnosis, and therapy. Circ Res 2017;121(07):749-770

6 Ammash NM, Seward JB, Bailey KR, Edwards WD, Tajik AJ. Clinical profile and outcome of idiopathic restrictive cardiomyopathy. Circulation 2000;101(21):2490-2496

7 Otto CM. Calcification of bicuspid aortic valves. Heart 2002;88 (04):321-322

8 Frohlich ED, Apstein C, Chobanian AV, et al. The heart in hypertension. N Engl J Med 1992;327(14):998-1008

9 Valentine RJ, Verstraete R, Clagett GP, Cohen JC. Premature cardiovascular disease is common in relatives of patients with premature peripheral atherosclerosis. Arch Intern Med 2000;160 (09):1343-1348

10 McCready RA, Vincent AE, Schwartz RW, Hyde GL, Mattingly SS, Griffen WO Jr. Atherosclerosis in the young: a virulent disease. Surgery 1984;96(05):863-869

11 Vakil K, Minami E, Fishbein DP. Right ventricular sarcoidosis: is it time for updated diagnostic criteria? Tex Heart Inst J 2014;41 (02):203-207

12 Topol EJ, Traill TA, Fortuin NJ. Hypertensive hypertrophic cardiomyopathy of the elderly. N Engl J Med 1985;312(05):277-283

13 Rai R, Swetha T. Association of anti-phospholipid antibodies with connective tissue diseases. Indian Dermatol Online J 2015;6(02): 89-91

14 Gunnarsson R, Hetlevik SO, Lilleby V, Molberg Ø Mixed connective tissue disease. Best Pract Res Clin Rheumatol 2016;30(01): 95-111

15 Chatterjee S, Flamm SD, Tan CD, Rodriguez ER. Clinical diagnosis and management of large vessel vasculitis: Takayasu arteritis. Curr Cardiol Rep 2014;16(07):499

16 Greco A, De Virgilio A, Ralli M, et al. Behçet's disease: new insights into pathophysiology, clinical features and treatment options. Autoimmun Rev 2018;17(06):567-575

17 Kobeissi E, Hibino M, Pan H, Aune D. Blood pressure, hypertension and the risk of abdominal aortic aneurysms: a systematic review and meta-analysis of cohort studies. Eur J Epidemiol 2019;34(06): 547-555 